\title{
The Human Exploration Telerobotics Project: Objectives, Approach, and Testing
}

\author{
Terrence Fong \\ Chris Provencher \\ Mark Micire \\ NASA Ames Research Center \\ Mail Stop 269-3 \\ Moffett Field, CA 94035 \\ terry.fong@nasa.gov
}

\author{
Myron Diftler \\ Reginald Berka \\ Bill Bluethmann \\ NASA Johnson Space Center \\ Mail Code ER 4 \\ Houston, TX 77058 \\ myron.a.diftler@nasa.gov
}

\author{
David Mittman \\ Jet Propulsion Laboratory \\ California Institute of Technology \\ 4800 Oak Grove Drive \\ Pasadena, CA 91109 \\ 818-393-0037 \\ david.s.mittman@jpl.nasa.gov
}

\begin{abstract}
In this paper, we present an overview of the NASA Human Exploration Telerobotics (HET) project. The purpose of HET is to demonstrate and assess how telerobotics can improve the efficiency, effectiveness, and productivity of human exploration missions. To do this, we are developing and testing advanced robots remotely operated by ground controllers on Earth and by crew on the International Space Station. The outcome of these tests will provide insight into the requirements, benefits, limitations, costs and risks of integrating advanced telerobotics into future human missions. In addition, the engineering data acquired during these tests will inform the design of future telerobotic systems.
\end{abstract}

\section{TABLE OF CONTENTS}

1. INTRODUCTION............................................... 1

2. GROUND CONTROL OPERATIONS ...................2

3. CREW CENTRIC OPERATIONS ......................2

4. CONCEPT OF OPERATIONS ........................... 3

5. RовоTS ............................................................ 4

6. Robot DATA COMMUNiCATIONS.................. 6

7. PROTOTYPE System TESTING ......................6

8. CONCLUSION .................................................. 8

ACKNOWLEDGEMENTS..........................................8 8

REFERENCES ........................................................ 8

BIOGRAPHIES ........................................................9

\section{INTRODUCTION}

Future human missions to the moon, Mars, and other destinations offer many new opportunities for exploration. However, crew time will always be limited and some work will not be feasible for astronauts to do manually [1]. Robots can complement human explorers, performing work under remote control from a crew vehicle, or even from Earth. A central challenge, therefore, is to understand how human and robot activities can be coordinated to maximize crew safety, mission success, and scientific return.

Robots can do a variety of work to increase the productivity of human explorers. Robots can perform tasks that are tedious, highly repetitive or long-duration, such as conducting site surveys. Robots can perform work that is beyond human capability, such as operating in dangerous environments and performing tasks that require great force. Robots can perform work ahead of humans, such as scouting, that help prepare for future manned activity and missions. Robots can also perform "follow-up" work after humans leave, completing tasks started by humans, or conducting supplementary tasks designated by humans.

The remote operation of many International Space Station (ISS) systems by ground control has become an accepted practice for certain tasks during the past decade. For robots, however, these tasks have been limited to positioning maneuvers of external payloads and structures using largescale manipulator arms. A key question, therefore, is whether more advanced ground control based telerobotic operations, involving a variety of robots and control modes, can yield tangible performance and productivity gains for both intravehicular activities (IVA) and extravehicular activities (EVA).

NASA and other space agencies are currently developing the architectures required for future space exploration [2][3]. Many of these architectures include the use of crewed, orbiting spacecraft and hypothesize that having astronauts remotely operate surface robots from these spacecraft is an effective way to perform certain exploration tasks [4]. However, this concept of operations has never been tested in space in a fully operational manner. Thus, it is not clear which technical and operational risks must be mitigated, nor which technology gaps must be closed to make this approach practical.

The key objective of the Human Exploration Telerobotics (HET) project, therefore, is to study how advanced remotely operated robots may increase the performance, reduce the costs, and improve the likelihood of success of future human exploration missions. To do this, we are developing, demonstrating and testing a variety of telerobotic systems, which can be operated by ground controllers on Earth and by crew in space.

Many of our tests make use of the ISS as a testbed. Although the logistics of testing on the ISS are complex (particularly in terms of certification, training, and crew scheduling), the ISS is the only facility available for performing high-fidelity simulations of future deep-space 
human missions. Ground-based simulators, in both laboratories and outdoor testbeds, lack fidelity in many areas. In particular, ground-based simulators fail to capture the effects of:

- micro-gravity on crew (this affects sensorimotor performance, etc.)

- long-duration stay in space on crew (this affects cognition, proficiency levels, etc.)

- crew activities, workload, and other sources of inflight stress

- flight vehicle constraints (including micro-gravity workspace, crew displays, etc.)

- operational complexity (particularly coordination with ground control, scheduling, etc)

Our testing falls into two broad categories: ground control operations and crew centric operations. Within these two categories, we are studying five prototype telerobotic systems: (1) IVA Dexterous Manipulator, (2) IVA FreeFlyer Mobile Sensor, (3) EVA Dexterous Manipulator (simulated), (4) EVA Free-Flyer Mobile Sensor (simulated), and (5) Crew Controlled Surface Telerobot.

\section{Ground Control Operations}

The central question we are addressing with ground control operations is: How can robots in space be safely and effectively operated from Earth to enable more productive human exploration? In other words, under what operational conditions and scenarios can robots be controlled by ground control to improve how crew explore and work in space?

To answer this question, we are maturing the capabilities of dexterous and human-safe robotics for use in space. We are also exploring the appropriate roles for robots, crew, and

Table 1. Ways in which robots can enable more productive crews during human exploration missions.

\begin{tabular}{|c|l|}
\hline \multirow{4}{*}{ Function } & \multicolumn{1}{c|}{ Roles } \\
\hline \multirow{4}{*}{$\begin{array}{c}\text { Offloading } \\
\text { Crew }\end{array}$} & EVA site setup and teardown \\
\cline { 2 - 3 } & ISS experiment inspection \\
\cline { 2 - 3 } & ISS science experiment manipulation \\
\cline { 2 - 3 } & Routine in-flight maintenance (IFM) \\
\cline { 2 - 3 } Crew & Non-routine maintenance \\
\cline { 2 - 3 } & Inventory management \\
\cline { 2 - 3 } & Manipulation of large payloads \\
\cline { 2 - 3 } & Remote camera for inspection \\
\cline { 2 - 3 } & Remote camera for situation awareness \\
\cline { 2 - 3 } & Working as a tool using crew apprentice \\
\cline { 2 - 2 } & Provide another “set of hands" \\
\hline
\end{tabular}

ground control in a variety of tasks. Our work focuses on robotic systems that have the potential to contribute significant gains in productivity and reduced operating costs to human exploration. Table 1 lists some of the ways in which robots can support crews in space.

\section{Ground Controlled Telerobots on Crew Vehicle}

Since crew time is always a premium in space, the best use of telerobotics may be as a means to offload mundane, repetitive, and routine work from humans. This would then enable crew to focus more of their time on tasks that require human cognition, dexterity, or involvement.

With the ISS, we are examining how ground-based personnel can remotely operate dexterous manipulators and free-flyer robots to perform a range of routine work inside crew vehicles:

- Air filter replacement. A dexterous robot with mobility capability can perform the highly repetitive tasks associated with maintaining equipment racks, such as routine air filter replacement.

- Experiment maintenance and monitoring. The numerous science experiments on the ISS require regular maintenance and visual monitoring by crew to replenish consumables and monitor progress. A dexterous IVA robot that can manipulate switches and image recording can perform many of these routine tasks.

- Inventory. A free-flyer robot equipped with appropriate image or proximity sensors, such as an RFID reader, can locate and identify items stowed aboard the ISS.

- Environmental survey. A free-flyer robot, equipped with suitable sensors, can roam the interior of the ISS to collect dosimetry data (lighting, sound, radiation, etc) to monitor environmental conditions and to detect anomalies.

\section{Crew Centric Operations}

The central question we are addressing with crew centric operations is: When is it worthwhile for astronauts to remotely operate surface robots from a flight vehicle during a human exploration mission? In other words, under what operational conditions and scenarios is it advantageous for crew to control a robot from a flight vehicle, habitat, etc. rather than a ground control team located on Earth?

To answer this question, we need to first define what we mean by "worthwhile." For human exploration, some important criteria are: (1) increasing crew productivity; (2) increasing crew safety; (3) reducing crew workload; (4) reducing dependency on consumables (fuel, oxygen, etc.); (5) reducing risk; (6) improving likelihood of mission success; and (7) improving science return. 
Depending on the mission (independent or as part of an extended campaign), each of these criteria may be significant. Moreover, the relative importance of each criterion will also depend on mission constraints, including duration, budget, and available resources (communications, power, etc).

We also need to understand the factors that vary from system to system, and from mission to mission. Clearly, the robot configuration (physical form, function, autonomy, sensors, etc.), control modes (rate control, interactive commanding, supervisory control, etc.), and user interface (for planning, commanding, monitoring, and analysis) all play a role. In addition, the characteristics of the communications link (bandwidth, latency, disruption tolerance, quality of service, etc) will have a major effect. Most importantly, the type and number of tasks that must be carried out will depend on the particular destination and objectives of each mission.

Finally, we should consider what is different about crew operating robots versus a team at mission control? The most obvious difference is the space environment, which affects human performance due to reduced gravity, radiation exposure, and other stress factors. Second, the number of work hours available for crew to operate robots is much less than for a ground team. This difference is due to having both limited mission "utilization" time and more personnel on the ground. Third, the communications link is different. In space, the crew can use a high-bandwidth, low-latency, direct connection to the robot, whereas mission control will have to rely on a low-bandwidth, high-latency link. Finally, crew will have fewer operational resources (processing, displays, etc.) than a ground team can use.

\section{Crew Controlled Telerobots on Crew Vehicle}

The same types of robots that are remotely operated by ground control (i.e., dexterous manipulators and free-flyers) can also be remotely operated by astronauts from within crew vehicle. This concept of operations is appropriate when: (1) poor, delayed, or intermittent communication prevents ground control from performing the task; (2) the crew's physical presence is critical to performing the task; or (3) local operations significantly outperforms remote operations (e.g., number of command cycles).

In HET we are assessing the trade-offs of crew controlling telerobots on ISS as compared to ground control. Our assessment focuses on simulating EVA tasks such as dexterous manipulation (routine maintenance and emergency repair) and mobile camera work (e.g., visual inspection surveys).

\section{Crew Controlled Surface Telerobots}

Another use case that we are studying is "crew controlled surface telerobots." In other words, the concept of crew remotely operating a robot (e.g., a planetary rover) deployed to a target surface (Mars or an asteroid) from a distant crew vehicle or habitat [4][5].
Surface robots remotely operated by crew can significantly enhance future exploration missions by working in a manner complementary to humans [6][7][8]. This is especially true when surface time is limited, or when astronauts are required to operate independently of ground control. Robots can be used to perform repetitive, time-consuming and other functions that are not necessary for humans to perform.

In our work, we are starting to examine how crew can remotely operate surface robots using a range of control modes, from direct teleoperation (position and rate control) to supervised autonomy (e.g., command sequencing with interactive monitoring). Crew will use surface robots to perform tasks such as:

- Mobile sensor platform. The robot will carry sensors (cameras, range sensors, etc.) to perform scouting, landing and docking site examination, etc. Surface robots can obtain data with significantly higher resolution than satellite-based instruments and at viewpoints not achievable from space.

- Dexterous mobile manipulation. The robot will be used to deploy payloads (e.g., instrument packages), to collect samples, retrieve equipment, or to close-out EVA tasks.

- Autonomous fieldwork. The robot will perform repetitive or long duration tasks required to characterize or to prepare a work site. Emphasis will be placed on performing tasks that would otherwise consume too much of crew surface time allocation, crew consumables, or pose significant risk (due to radiation exposure, terrain hazards, etc).

- Real-time support. The robot will be used to perform tasks requiring real-time decision-making and timecritical response. Emphasis will be placed on tasks that require high-bandwidth, low-latency data communications, such as real-time camera views to support EVA, emergency response, etc.

\section{Concept of Operations}

To date, NASA mission operations have focused on different concepts of operation for human and deep space robotic programs [9]. Human missions to Low Earth Orbit (LEO) and the Moon are characterized by near-continuous communication with minimal delay (i.e., less than a few seconds). Mission control and astronauts in space work together as an integrated team, performing tasks and resolving problems in real-time.

In contrast, deep space robots (orbiters, landers, and planetary rovers) must function independently for long periods of time without communication to ground controllers. Consequently, mission operations have traditionally centered on the use of carefully scripted and validated command sequences, which are intermittently uplinked to the robot for independent execution [10]. 
Future deep-space human missions, however, will need to combine aspects from both concepts of operations. Moreover, missions that combine human and robotic activity will need to consider operational constraints due to location (in-space or on-surface), communication link (bandwidth, latency and availability), and timelines (strategic, tactical, execution), all of which may vary - even during a single mission.

In this project, we are studying the following concepts of operations for telerobotic operations:

- Crew centric. The crew performs planning, operations, contingency handling and analysis. Ground control supports crew on an intermittent and timedelayed basis. This concept of operations is appropriate when conditions (orbital geometry, time-delay, etc.) make it impractical for ground control to remotely operate robots.

- Crew/ground shared. Ground control performs planning and analysis from Earth. The crew performs tactical ops. This concept of operations enables many robot command cycles to be performed, even when the robot is far from Earth.

- Ground centric. Ground control performs planning, operations, and analysis from Earth. The crew performs interventions when needed. This concept of operations is well suited for handling contingencies that are beyond robot autonomy capabilities.

It is important to note that on the ISS, and likely during future human missions, the crew's activity schedule consists of many varied activities, most of which are planned (scheduled) in advance. Thus, whenever crew is required to perform telerobotic operations, particularly unplanned interventions, they will have to context switch. Consequently, an important objective in our tests is to understand how task switching affects human-robot operations performance as well as crew efficiency and productivity.

\section{RoBOts}

A number of free-flyer platforms have already been remotely operated at the ISS, including the AERCam Sprint Free-Flyer [11] in EVA and the SPHERES [12] satellites in IVA. In addition, manipulators, including the SSRMS and SPDM, have been used to position payloads in EVA. To date, however, no semi-autonomous robot has yet been operated while in close proximity to crew.

Thus, a key objective of HET is to demonstrate how robots can be used to perform a wide range of human exploration tasks. To do this, we are making use of robots capable of IVA work (including dexterous manipulation and mobile imaging), simulated EVA work outside of a crew vehicle, and surface work (survey, scouting, etc.) on unstructured, natural terrains.

\section{ATHLETE}

ATHLETE is a six-limbed, hybrid rolling and walking robot. ATHLETE is capable of rolling over relatively flat terrain and walking over extremely rough or steep terrain. Each limb has 6 degrees-of-freedom (DOF) and is equipped with a motorized wheel. The first generation ATHLETE robots were constructed in 2005 and the current generation robots were built in 2009 .

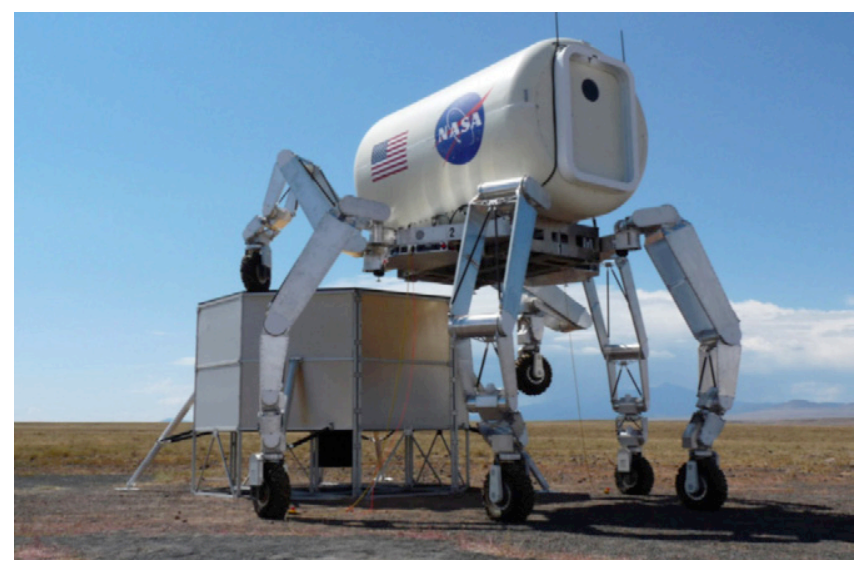

Figure 1. The "T12" ATHLETE robot stepping off of a simulated lander.

The current ATHLETE, called "T12", is implemented as a pair of three-limbed halves, each of which is called a "TriATHLETE." Each Tri-ATHLETE can be independently controlled, which gives the system tremendous modularity and flexibility for supporting the transport and positioning of large cargo and payloads.

The T12 ATHLETE stands to a maximum height of just over 4 meters. The robot has a total of 42 DOF (six legs with 7 DOF each), is equipped with numerous sensors (cameras, force sensors, and joint encoders), and has a payload capacity of $450 \mathrm{~kg}$ in Earth gravity.

\section{K10 Planetary Rover}

The K10 planetary rover (Figure 2) is designed to operate on planetary surfaces as a mobile instrument platform [1]. K10 has four-wheel drive and all-wheel steering with a passive, differential averaging suspension. This design allows operation on moderately rough natural terrain. K10 can automatically avoid obstacles while driving long distances and over a wide variety of terrain.

$\mathrm{K} 10$ is equipped with a variety of navigation and positioning sensors (hazard cameras, 2D lidar, suntracker, differential GPS, inertial measurement unit, and digital compass) and instruments (3D lidar, panoramic cameras, ground penetrating radar, percussive penetrometer, spectrometers, etc.). K10 has been used at numerous planetary analog sites (including Haughton Crater, Moses Lake Sand Dunes, and Black Point Lava Flow) to perform work both before, and after, human activity [6][7][8]. 


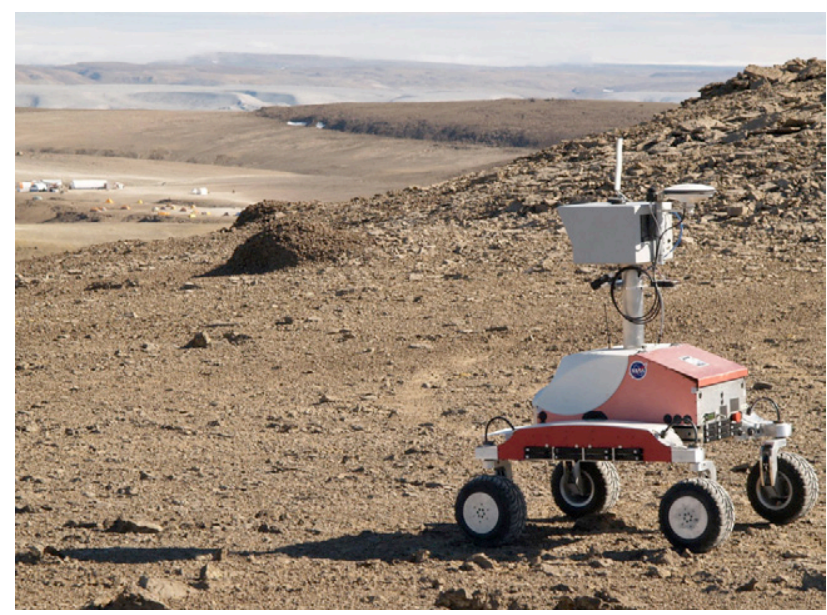

Figure 2. K10 rover at Haughton Crater (Canada).

Each K10 has four-wheel drive and all-wheel steering with a passive, averaging rocker suspension. This design allows operation on moderately rough natural terrain at human walking speeds (up to $90 \mathrm{~cm} / \mathrm{s}$ ). K10's software system is based on a Service-Oriented Architecture, which includes modules for locomotion, navigation, and instrument data acquisition.

\section{Robonaut 2}

Robonaut 2 (R2) is a two-arm humanoid robot (Figure 3) that is the latest result of a long-term NASA effort to develop robots that have manipulation capabilities similar to suited astronauts [13]. In the future, dexterous robots will be able to make use of astronaut hand tools without modification and to perform EVA work to help reduce the amount of consumables used during human missions.

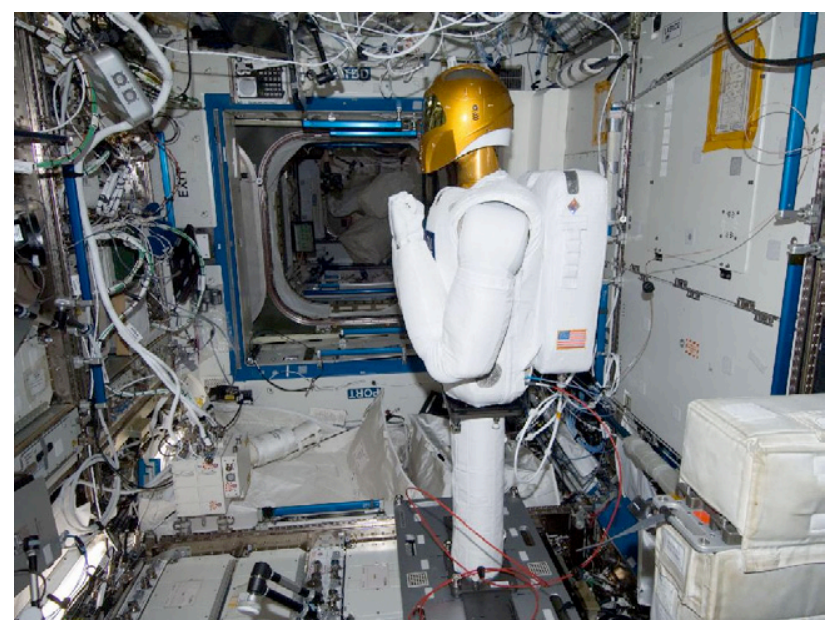

Figure 3. Robonaut 2 in the International Space Station.

R2 has a total of $42 \mathrm{DOF}$, including two 7 DOF arms, two 12 DOF hands, a 3 DOF neck, and a 1 DOF waist [14]. R2 also includes integrated avionics (computing, power conditioning, etc.) and numerous distributed sensors (cameras, 3D lidar, force/torque, etc). R2 uses a dualpriority impedance controller to manage forces in both operational and joint space [13].
R2 was delivered to the ISS in February 2011 on the Space Shuttle Discovery STS-133 flight. Initial power-on testing with R2 took place in August 2011. Preliminary check-out of robot systems and safeguarded motions began in September 2011. During the first phase of R2's deployment on the ISS, it will be used as an IVA robot on a fixed base. Future enhancements to the system may include IVA mobility, or even possibly EVA versions.

\section{SPHERES}

The Synchronized Position Hold Engage and Reorient Experimental Satellites (SPHERES) are volleyball-sized free-flyers that have been on the ISS since 2006 [12]. SPHERES were originally developed by the Massachusetts Institute of Technology to serve as a platform for testing spacecraft guidance, navigation, and control (GN\&C) algorithms in a micro-gravity environment. To date, astronauts have conducted more than 25 test sessions with individual and multiple SPHERES to study formation flying, rendezvous, and docking.
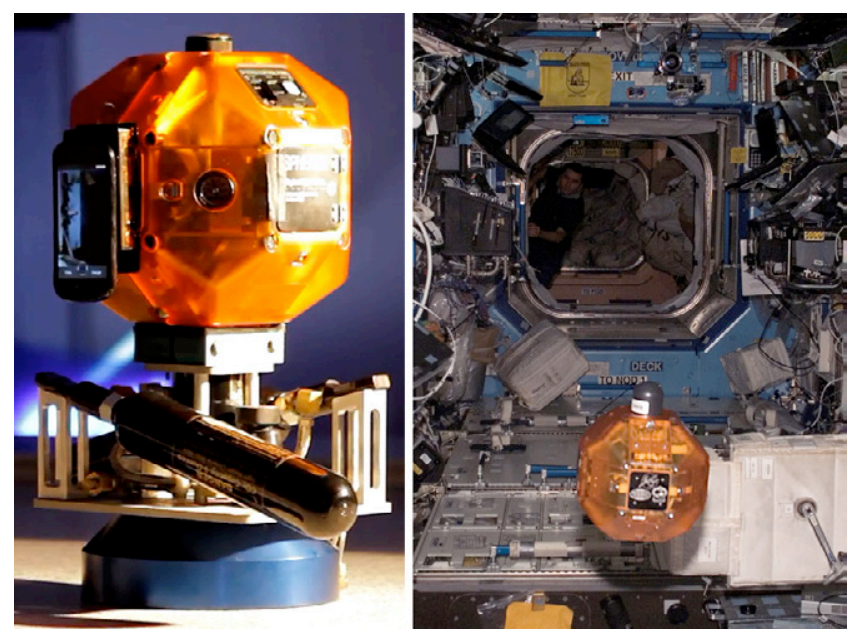

Figure 4. SPHERES equipped with an Android smartphone upgrade.

Each SPHERE is fully self-contained with propulsion, power, computing and navigation equipment. A cold-gas (carbon dioxide) thruster system is used for motion control and DC batteries provide electronic power. For processing, the SPHERES rely on a digital signal processor, which handles all on-board software functions including navigation, device control, and communications. An external ultrasonic, local positioning system provides data to estimate the position and orientation of SPHERES.

Because the SPHERES were originally designed as a GN\&C testbed, they require some upgrades in order to be useful as remotely operated robots. The primary change that we are making is to install an Android-based smartphone as a computing upgrade. The smartphone provides SPHERES with a high-performance processor (including graphical processing unit), color cameras, additional sensors (temperature, ambient light, sound), and high-bandwidth wireless networking for data communications. 


\section{Robot Data Communications}

Modern robots are highly complex systems. Consequently, the software for these robots must be implemented as multiple modules (perception, navigation, operator controls, etc) by multiple developers, who often work in a distributed team. To facilitate the integration of independently developed modules into fielded systems, as well as to encourage interoperability, reusability, and maintainability, a robotics software framework is required.

The Robot Application Programming Interface Delegate (RAPID) is an open-source framework for remote robot operations [15]. RAPID is designed to: facilitate integration of experimental robot software modules created by a distributed development team; improve the compatibility and reusability of robotic functions; and speed prototype robot development in a wide range of configurations and environments [16]. RAPID includes a standard programming interface and data distribution middleware.

RAPID includes the following components:

- The RAPID Language. An "application programming interface" (API) that defines an interface for accessing and distributing data between robots, operator interfaces, and payloads. The RAPID Language is similar in functionality to the CLARAty [17] communications interface and the JAUS [18] message set, but addresses the operational needs of exploration fieldwork (site surveys, payload transport, etc) and supervisory telerobotics over time-delay.

- The RAPID Workbench. A software tool that enables an operator to command and monitor robot modules without requiring detailed knowledge of the underlying implementation. The RAPID Workbench sends and receives messages that are in the RAPID language. The RAPID Workbench is implemented in the open-source Eclipse software framework.

- The RAPID Middleware. A system for distributing data between robot modules. The RAPID Middleware is designed to be language independent (i.e., client software can be written in different languages but can interoperate) and support multiple platforms. The underlying data transport is intended to be "pluggable" (i.e., can be easily replaced).

- The RAPID Bridge. A reference design for translating RAPID Language messages into robot-specific data. Example implementations have been developed for the ATHLETE, K10 planetary rover, and other NASA robots.

For HET, we are using RAPID to support remote operations of the ATHLETE, K10, and SPHERES robots. In particular, we use RAPID for robot commanding (primitive actions and command sequences), for monitoring (telemetry including robot state, position, task progress, etc), and transfer of large-volume datasets (e.g., panoramic image sets).

We expect that the results and lessons learned from our ISS tests, will inform future refinements and extensions to RAPID. These include: latency mitigation, delay and disruption tolerant data messaging, transfer of control and coordination mechanisms, and interface refinements. In addition, we anticipate that our use of RAPID in an operational flight environment may lead to its adoption as an open, interoperable standard for space telerobotics.

\section{Prototype System Testing}

The functional space of telerobots is extremely large, especially when considering all the systems that might be deployed on planetary surfaces, to small bodies (e.g., nearEarth asteroids), and in-space. Because this space is so broad, it is highly unlikely that there exists an "optimal" system configuration.

Thus, to explore and demonstrate different aspects of telerobotics for human exploration, we are testing five different prototype systems. We chose these systems based on their potential to increase performance, reduce cost, and improve the likelihood of success of future missions.

\section{IVA Dexterous Manipulator}

The "IVA Dexterous Manipulator" is used to demonstrate that ground control can perform IVA tasks that require dexterous manipulation. The primary objective is to assess fine manipulation capabilities and the use of crew tools. In particular, we are studying how primitive actions, such as switch closures, can be performed in a reliable and humansafe manner without artificial fixtures.

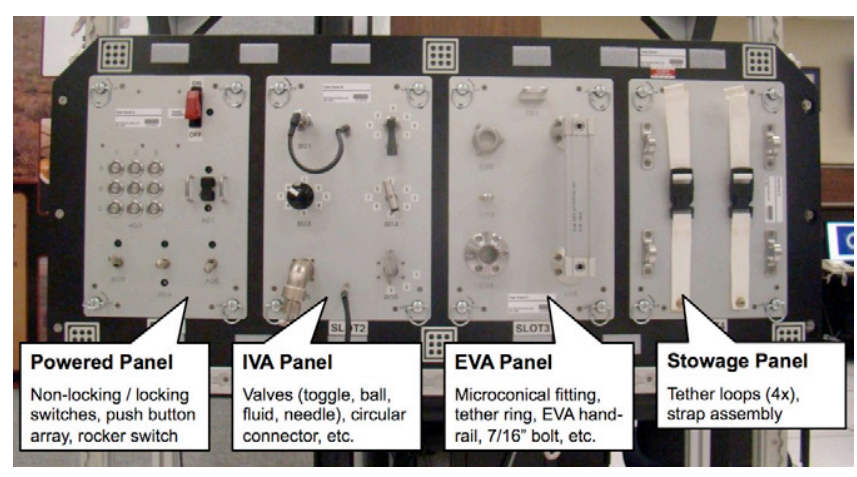

Figure 5. Modular task board for manipulation testing.

Figure 5 shows the modular task board that is used for these tests. The left-most section, the "Powered Panel", contains an array of locking and non-locking switches, push buttons, and rocker switches. We use this panel to assess the system's ability to manipulate switches. The center-left section, the "IVA Panel", contains a variety of valves (toggle, ball, fluid, needle) and connectors. We use this panel to assess the system's ability to work with more challenging IVA components. 
During our testing, we record a variety of data to characterize robot performance and to identify areas for improvement. This data includes joint positions, force/torque, primitive action success, task times, etc.

\section{IVA Free-Flyer Mobile Sensor}

The "IVA Free-Flyer Mobile Sensor" is used to demonstrate that ground control can perform IVA tasks, which require mobile sensors and which would normally have required crew. These tasks include interior environmental surveys (radiation, sound levels, etc.) and mobile camera work (e.g., video documentation and "fly throughs"). To test this prototype system, we use an upgraded SPHERES to perform light level and ambient temperature survey within an ISS module.

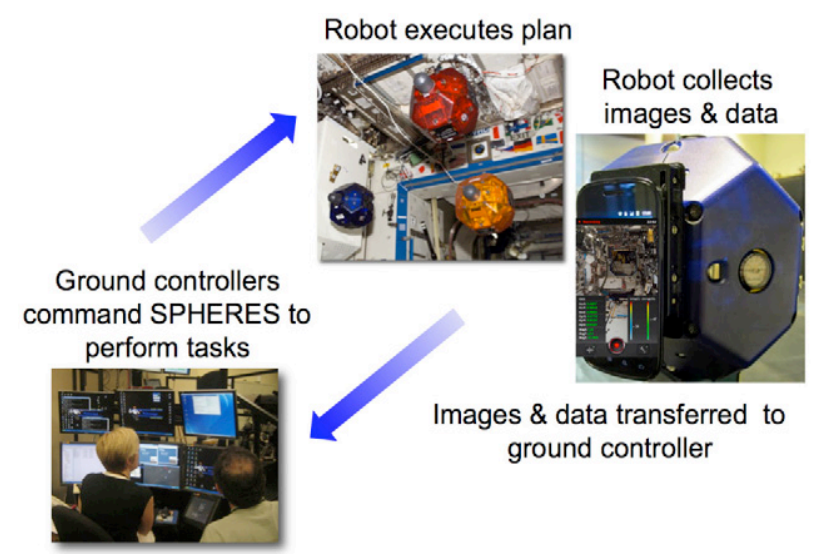

Figure 6. IVA Free-Flyer Mobile Sensor system concept.

A key part of our testing is to characterize ground control operations of SPHERES (Figure 6). To do this, we record a variety of time-stamped data:

- SPHERES position, power, and health

- Survey instrument data (e.g., light level) temperature, etc.)

- Commanded plans versus actual executed plans

- Data transfer statistics (bandwidth, latency, jitter)

We then use this data to calculate several human-robot interaction metrics, such as Mean-Time-BetweenInterventions (MTBI) and Mean-Time-CompletingInterventions (MTCI) [19]. We also use critical incident technique to analyze ground controller work activity and to identify significant problems during task performance.

\section{EVA Dexterous Manipulator (simulated)}

The "EVA Dexterous Manipulator" is used to simulate a system that crew might one day use to remotely perform work outside of a flight vehicle. The primary goal with this prototype is to identify issues associated with crew centric operations of a dexterous manipulator. In this test, crew on ISS remotely operates Robonaut 2 to perform simulated EVA work.
Figure 5 shows the modular task board that is used for these tests. The center-right section, the "EVA Panel", contains a microconical fitting, tether ring, and EVA handrail. We use this panel to assess the system's ability to work with artifacts commonly encountered during EVA.

Because EVA work would be performed in close proximity to a flight vehicle, we expect there would be little (or no) communications delay between the crew and robot. Thus, unlike ground operations of the "IVA Dexterous Manipulator", crew is able to make use of control modes and displays (e.g., head-mount stereo) that afford real-time operation.

\section{EVA Free-Flyer Mobile Sensor (simulated)}

The "EVA Free-Flyer Mobile Sensor" is used to simulate a system that crew might one day use to remotely perform work outside of a flight vehicle. The primary goal with this prototype is to identify issues associated with crew centric operations of a robotic free-flyer that carries short-range sensors (e.g., for proximity operations outside a spacecraft). In this test, crew on ISS remotely operates an upgraded SPHERES to perform a simulated, routine EVA visual inspection of an external structure.

In contrast to the "IVA Free-Flyer Mobile Sensor", this test focuses on a single operations mode: supervisory control of autonomous robot operations. Emphasis is placed on examining how the crew can maintain situation awareness when they are only intermittently interacting with the robot, as well as how effectively they can intervene to cope with contingencies. To do this, we record the following data:

- SPHERES position, power, and health

- Inspection camera images

- Situation awareness (Situational Awareness Global Assessment Technique [20])

- User interface: control mode changes, data input, button clicks

- Commanded plans versus actual executed plans

We also apply critical incident technique and heuristic evaluation to identify operational and usability issues.

\section{Surface Telerobots}

The "Surface Telerobots" test is used to simulate a future deep-space human mission, during which crew remotely operates robots on a planetary surface from inside a flight vehicle (Figure 7). The primary goal is to study humanrobotic system operation, with emphasis on confirming the issues identified and validating the lessons learned from prior analog field tests [6][7][8]. In this test, a single astronaut on the ISS remotely operates the K10 planetary rover and ATHLETE robot to perform landing site evaluation (slopes, hazards, etc) and traverse scouting (accessibility, hazards, etc). 

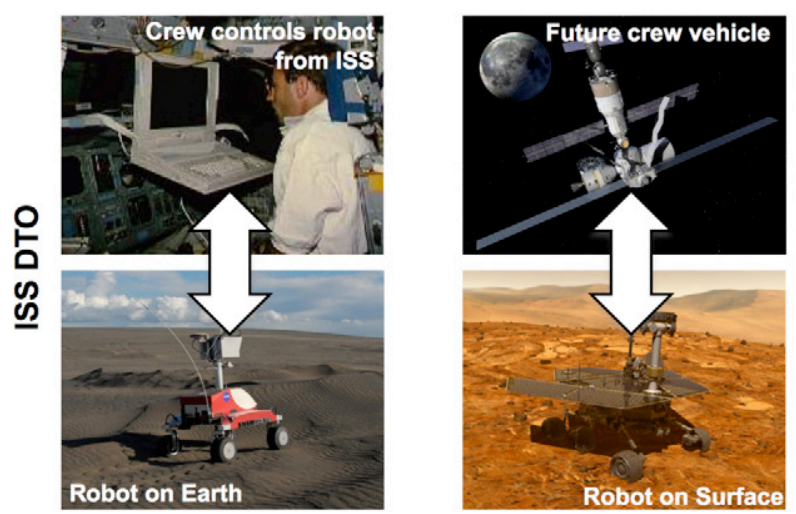

Figure 7. Surface Telerobots system concept.

The objectives of this test are to evaluate how crew can effectively control the robot, to ascertain the level of situation awareness attained and maintained, and to assess the quality of the sensor data obtained. Multiple control modes (ranging from position/rate control to supervisory control) and concept of operations (crew centric, crew/ground shared, and ground centric) are all employed.

During testing, we record the following data:

- Data communication: data transfers, delay, message rate, bandwidth required

- Robot telemetry: position and orientation, power, health, instrument use

- User interface: control mode changes, data input, button clicks

- Operations: sequence generation statistics, task success/failure

- Crew questionnaires: workload, situation awareness

We then employ a variety of metrics to assess system operation, including "productive time" [21], Situational Awareness Global Assessment Technique (SAGAT) [20], and NASA Task Load IndeX (TLX) [22]. In addition, Critical Incident Technique is used to identify major problem areas and develop strategies for improving system performance.

\section{Conclusion}

The Human Exploration Telerobotics project is developing, demonstrating, and testing how advanced, remotely operated robots may increase the performance, reduce the costs, and improve the likelihood of success of future human exploration missions. We expect our work will help mitigate risk by testing and proving methodologies to be used in future human and robotic missions. In addition, the results of our tests will inform the development of new design reference missions, help to evolve approaches to human controlled robotics, and enable new ways to explore space with humans and robots.

\section{ACKNOWLEDGEMENTS}

We thank Chris Moore for his unwavering support and leadership that created the HET project. We also thank Jason Crusan, Bruce Yost, Andres Martinez, Steve Ormsby, Melissa Boyer, and Alvar Saenz-Otero for supporting SPHERES. We also thank Ernie Smith, Tim Kennedy, and the JSC Mission Operations Directorate for their assistance with ISS operations.

The NASA Enabling Technology Development and Demonstration program, the NASA Technology Demonstration Missions program, and the International Space Station program sponsored this work.

\section{REFERENCES}

[1] Fong, T., Bualat, M., et al. 2008. "Field testing of utility robots for lunar surface operations". AIAA2008-7886. In Proceedings of AIAA Space 2008.

[2] Augustine, N., et al. 2009. Review of the U.S. Human Spaceflight Plans Committee: Seeing a Human Spaceflight Program Worthy of a Great Nation.

[3] International Space Exploration Coordination Group. 2011. The Global Exploration Roadmap.

[4] Nergaard, K., et al. 2009. METERON: An Experiment for Validation of Future Planetary Robotic Missions. CDF Study Report. CDF Study Report: CDF-96(A).

[5] Dupuis, E., Langlois, P., et al. 2010. "The AvatarEXPLORE Experiments: Results and Lessons Learned". In Proceedings of the International Symposium on Artificial Intelligence, Robotics, and Automation in Space.

[6] Deans, M., Fong, T., et al. 2009. "Robotic scouting for human exploration”. AIAA-2009-6781. In Proceedings of AIAA Space 2009.

[7] Fong, T., Abercromby, A., et al. 2009. "Assessment of robotic recon for human exploration of the Moon". IAC-09-A5.2-B3.6.7. In Proceedings of the 60th International Astronautical Congress.

[8] Fong, T., Bualat, M., et al. 2010. "Robotic follow-up for human exploration". In Proceedings of AIAA Space 2010.

[9] Mishkin, A., Lee, Y., Korth, D., and LeBlanc, T., 2007. "Human-Robotic Missions to the Moon and Mars: Operations Design Implications". In Proceedings of the IEEE Aerospace Conference.

[10] Mishkin, A., Limonadi, D., Laubach, S., and Bass, D. 2006. Working the Martian Night Shift: The MER Surface Operations Process. IEEE Robotics Automation Magazine.

[11] Williams, T. and Tanygin, S. 1998. On-orbit Engineering Test of the AERCam Sprint Robotic Camera Vehicle. Advances in the Astronautical Sciences 99(2).

[12] Miller, D., Saenz-Otero, A., et al. 2000. SPHERES: A Testbed for Long Duration Satellite Formation Flying in Micro-Gravity Conditions. Advances in the Astronautical Sciences 105. 
[13] Diftler, M., Mehling, J., et al. 2011. "Robonaut 2: the First Humanoid Robot in Space". In Proceedings of the IEEE International Conference on Robotics and Automation.

[14] Hart, S., Yamokoski, J., and Difter, M. 2011. "Robonaut 2: A New Platform for Human-Centered Robot Learning". In Proceedings of Robotics: Science and Systems.

[15] Robot Application Programming Interface Delegate (RAPID). 2009. Open-Source release v1. ARC-163681. NASA.

[16] Torres, R., Allan, M., Hirsh, R., and Wallick, M. 2009. "RAPID: Collaboration Results from Three NASA Centers in Commanding/Monitoring Lunar Assets. In Proceedings of the IEEE Aerospace Conference.

[17] Nesnas, I. 2007. The CLARAty Project: Coping with Hardware and Software Heterogeneity. In Brugali, D. (ed). Software Engineering for Experimental Robotics. Springer Tracts in Advanced Robotics.

[18] JAUS Core Service Set. 2010. SAE Standard AS5710. SAE International.

[19] Shah, J., Saleh, J., and Hoffman, J. 2008. Analytical Basis for Evaluating the Effect of Unplanned Interventions on the Effectiveness of a Human-Robot System. Reliability Engineering and System Safety 93.

[20] Endsley, M. 2000. Direct Measurement of Situation Awareness: Validity and Use of SAGAT. In M. Endsley and D. Garland (Eds.), Situation awareness analysis and measurement. Lawrence Erlbaum Associates.

[21] Schreckenghost, D., Milam, T., and Fong, T. 2010. Measuring Performance in Real-Time During Remote Human-Robot Operations with Adjustable Autonomy. IEEE Intelligent Systems 25(5).

[22] Hart, S. 2006. "NASA-Task Load Index (NASATLX); 20 Years Later". In Proceedings of the Human Factors and Ergonomics Society 50th Annual Meeting.

[23] Flanagan, J. 1954. The Critical Incident Technique. Psychological Bulletin 51(4).

\section{BIOGRAPHIES}

Terrence Fong is the Director of the Intelligent Robotics Group at the NASA Ames Research Center. He was formerly the deputy leader of the Virtual Reality and Active Interfaces Group at the Swiss Federal Institute of Technology / Lausanne. He received his B.S. and M.S. in aeronautics and astronautics from the Massachusetts Institute of Technology and his Ph.D. in robotics from Carnegie Mellon University.
Chris Provencher is a project manager in the Intelligent Robotics Group at the NASA Ames Research Center. He previously worked in the NASA Constellation programs. Prior to that he was an avionics instructor for the International Space Station program. He received a B.S. in electrical engineering from the University of Houston and a M.S. in computer engineering from the University of Houston / Clear Lake.

Mark Micire is a senior research scientist in the Intelligent Robotics Group at the NASA Ames Research Center and is the HET technical lead for SPHERES development and testing. He was formerly the president and CEO of American Standard Robotics. He received his B.S. and M.S. in computer science from the University of South Florida and his Ph.D. in computer science from the University of Massachusetts Lowell.

Myron Diftler is a senior engineer in the Software, Robotics and Simulation Division at the NASA Johnson Space Center. He is also the manager of the Robonaut 2 project. He received his B.S.E in mechanical engineering from Princeton University, his M.S. in electrical engineering from Yale University and his Ph.D. in mechanical engineering from Rice University.

Reginald Berka is a senior engineer in the Software, Robotics and Simulation Division at the NASA Johnson Space Center. He received his B.S. in mechanical engineering from Wichita State University and his Ph.D. in mechanical engineering from Rice University.

Bill Bluethmann is the Deputy Branch Chief of the Robotic Systems Technology Branch in the Software, Robotics and Simulation Division at the NASA Johnson Space Center. He is also the project manager of the NASA Human-Robotics Systems project. He received his Ph.D. in mechanical engineering from the University of Kansas.

David Mittman is a Senior Member of Technical Staff, Planning Software Systems, at the Jet Propulsion Laboratory. He is the HET technical lead for the Robot Application Programming Interface Delegate (RAPID) software. He is also the task manager for human-system interaction within the NASA Human-Robotic Systems project and oversees the implementation of new operations technologies for JPL's ATHLETE robot. He received a B.A. in psychology from Occidental College. 